\title{
A Sun compass in monarch butterflies
}

- ach autumn, monarch butterflies - (Danaus plexippus) migrate up to $4,000 \mathrm{~km}$ from breeding grounds in the eastern United States and Canada to overwintering sites in Mexico ${ }^{1,2}$. We tested the ability of monarchs to orient using a Sun compass by clock-shifting ${ }^{3}$ the butterflies and observing the orientation of their subsequent flight. Clock-shifted migrants reoriented their bodies in the predicted clockwise direction whereas control groups of migrants did not. This indicates that monarch butterflies are able to orient during their transcontinental migration using a Sun compass.

In conjunction with a knowledge of the time of day, many animals are able to use the Sun's changing azimuth to orient their migratory and homeward movements ${ }^{4}$. Beginning with Schmidt-Koenig ${ }^{3}$, Sun compass use has been demonstrated by clockshifting experiments. A change in the internal clock causes animals to misinterpret the position of the Sun, and hence change their direction of movement in a predictable way. In the Northern Hemisphere, for example, time-delayed migrants exhibit a clockwise change in orientation.

During September 1996, in eastern Kansas, we experimentally clock-shifted (delayed) migrating monarchs by six hours to determine whether these butterflies use a time-compensated Sun compass. We released clock-shifted subjects individually and watched them for $1-5 \mathrm{~min}$. We ran behind and beneath butterflies to estimate body orientations (headings) maintained for $15 \mathrm{~s}$ or more, using hand-held compasses. Migrants that were kept in the laboratory but not clock-shifted served as a sham control group and naturally migrating butterflies served as 'natural' controls.

The mean heading for clock-shifted butterflies was toward the west-northwest (Fig. 1a), which is $75^{\circ}$ from the mean southsouthwest orientation of the sham controls

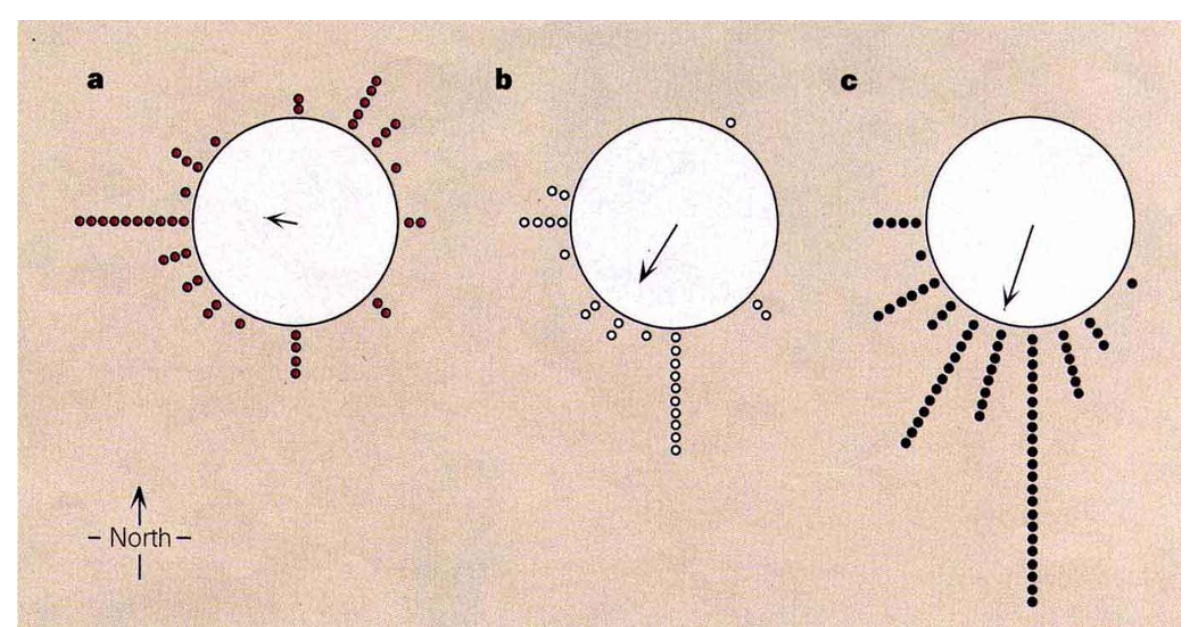

Figure 1 Mean body orientation data, giving resultant vector direction $(\mu)$ and length $(r)$ for a, clockshifted migrants $\left(\mu=287^{\circ} ; r=0.29 ; n=43\right.$, each circle represents 1 migrant $)$; b, sham controls $\left(\mu=211^{\circ} ; r=0.67 ; n=25\right.$, each circle represents 1 control); and c, natural controls $\left(\mu=200^{\circ}\right.$; $r=0.86 ; n=204$, each circle represents 3 controls). Subjects not exhibiting directional flight for any reason (for example, spiralling upwards on thermal currents, stopping to feed) were excluded from the analyses. Of 532 migrants observed, 272 yielded stable heading data.

(Watson's $F$-test, $F=13.84, P<0.01$; Fig. 1b) and $85^{\circ}$ from the mean natural control measure $(F=49.02, P<0.01$; Fig. $1 \mathrm{c})$. The mean heading of the clock-shifted butterflies bears out the clockwise change in direction predicted by the clock-shift delay experienced, and is of the magnitude predicted by the 6-hour time shift. Importantly, the two control groups exhibited strikingly similar mean headings $(F=1.93$, $P>0.05)$, indicating that there was no significant effect of captivity.

Monarch butterflies in North America use a Sun compass during their southward autumn migration, orienting their bodies using the time of day and the position of the Sun. The monarch butterfly thus joins the small group of species for which a Sun compass orientation mechanism has been demonstrated experimentally. In the absence of celestial cues on overcast days, however, monarchs still manage to orient towards the south-southwest ${ }^{5}$, suggesting that they also have a non-celestial backup mechanism of orientation, such as a geomagnetic compass ${ }^{6,7}$.

\section{Sandra M. Perez}

Department of Ecology and Evolutionary Biology,

University of Arizona,

Tucson, Arizona 85721, USA

e-mail:mperez@u.arizona.edu

Orley R. Taylor

Rudolf Jander

Department of Entomology,

University of Kansas,

Lawrence, Kansas 66045, USA

\section{Electrical recording from}

\section{a glass sponge}

Sponges arose very early in metazoan evolution. They do not have a nervous system, but some respond to stimulation by producing local contractions and one group, the 'glass sponges' (Hexactinellida), shows coordinated arrests of movements of the flagella, which produce the feeding current $^{1,2}$. We show here that these arrests are coordinated by propagated electrical impulses. This is, to our knowledge, the first report of electrical signalling in any sponge.

Previous attempts to record electrical activity from sponges have been hampered by the delicate, porous nature of the tissues, which makes attachment or insertion of electrodes difficult. In the sponge that we studied (Rhabdocalyptus dawsoni) the tissues are very flimsy, consisting of thin, perforated sheets and filamentous strands draped around a scaffold of spicules, like a three-dimensional cobweb $^{3}$. Few of the strands exceed $20 \mu \mathrm{m}$ in thickness and the surface layers are about $1 \mu \mathrm{m}$ thick.

To record from the sponge we dissociated tissue and allowed it to aggregate, using
Concanavalin A to promote adhesion ${ }^{4,5}$. When the aggregates had rounded off, we placed them on the surface of slabs of body wall taken from the original sponge. After $12 \mathrm{~h}$ the grafts were attached and after $24 \mathrm{~h}$ cytoplasm could be seen streaming into the sponge (Fig. 1a). The tissue formed a substantial lump to which suction electrodes could be attached (Fig. 1b).

After $24 \mathrm{~h}$, we put the slabs into a perfusion chamber in a shielded recording cage, inserted stimulating electrodes and positioned a thermistor-bead flow meter over the preparation to record changes in water flow across the body wall. We then attached 